\section{Prospects for New Vaccines against Tuberculosis}

\author{
Lise Brandt and Ian Orme \\ Colorado State University, Fort Collins, \\ CO, USA
}

\section{INTRODUCTION}

Tuberculosis kills more than 2 million people per year. In addition, the incidence of over 8 million new cases each year and the fuel added to the fire by the HIV epidemic lead to the concern that this global problem may be spiraling out of control. No new vaccine has been introduced for 80 years and no new (specifically approved) drug for more than 30 years. In both cases, the development of new preventions or treatments is a complex and difficult task that is further compounded by the fact that clinical trial assessments will be a lengthy business. If there is a glimmer of hope, then new advances in proteomic- and genomic-based technologies may accelerate both the identification of key antigens that can be used in new vaccines and the identification of bacterial biosynthetic pathways that can be used in drug discovery. This brief review discusses current advances in the preclinical screening of new vaccine candidates.

\section{THE EXISTING BCG VACCINE}

The existing vaccine for tuberculosis is called BCG, which stands for Mycobacterium bovis bacillus CalmetteGuerin after the two French scientists who derived this live vaccine from a culture of virulent bovine tuberculosis from 1908 to 1921 . Since then, more than a billion doses of BCG have been given. The vaccine is safe and inexpensive to produce.

But does it work? Whereas it clearly had a protective effect in certain controlled clinical trials, in others its effects were low or even zero $(8,11,12,23)$. This is a complicated matter to explain, and for that matter still contentious, but can be summarized as follows. BCG does have a protective effect if given to young children, but its activity diminishes as the individual gets older (into the teenage years), and it does not appear to work if given to adults. The vaccine can be seriously inhibited if given to people already sensitized to environmental mycobacteria such as M. avium $(7,25)$. Moreover, the BCG parent strain has been widely distributed around the world over the past 80 years and, in the process, resulting in the suspicion that the vaccine has been "attenuated to impotence" (5). This suspicion has been confirmed at the molecular level by the demonstration that daughter strains of BCG have clearly lost more and more genetic content (4).
While there is a general consensus that a new vaccine needs to be developed, there is no consensus as to whether this vaccine should replace BCG or be used to boost it. Of course, this depends on how good a new vaccine might turn out to be. Because of the time needed to evaluate new vaccines at the clinical level, it will probably be wise to look at the feasibility of both approaches. In addition, since BCG does seem to confer protection against disseminated and meningeal tuberculosis in children, as well as having a protective effect against leprosy, then it is very unlikely to be completely replaced any time soon.

\section{NEW VACCINE APPROACHES}

Preclinical testing of new vaccines for tuberculosis currently falls into four steps. First, new candidates are tested in mice to ensure that they are capable of inducing a TH1 response that is necessary for the efficient generation of protective immunity to tuberculosis (26). The next step is to immunize mice and then (usually a month later) challenge them with a low-dose aerosol challenge infection with M. tuberculosis. Then, 30 days later, the mouse is euthanized and the bacterial load in the lungs determined, as well as an assessment of any potential changes in the lung gross pathology. If there is a statistically significant reduction in this level in the vaccinated mice, then the vaccine candidate can be regarded as active. This is not a perfect model; it gives no indication of the longevity of the vaccine effect or any longer-term positive changes in pathology, but it is cost effective as a model, allowing relatively large numbers of candidates to be screened. In this regard, to date the NIH program at Colorado State University (CSU) has tested more than 220 candidates.

The third level is to use the guinea pig to further test the efficacy of the vaccine. This animal is exquisitely sensitive to M. tuberculosis infection (the lethal dose is probably less than five), and it exhibits a pathological process in the lungs that have several similarities to that seen in humans with tuberculosis. As a result, it is considered by many to be perhaps the most convincing model in which vaccine activity can be assessed. A drawback, however, is that it requires considerable animal housing space and is expensive to keep, reducing its practicality.

Protection in the guinea pig can be demonstrated in two ways. The first is to use the same read-out as the mouse model and look at day 30 lung burden (21). The second, pioneered at CSU, is to follow long-term survival and attendant lung immunopathology (2). Unfortunately, a limitation of the guinea pig model is the lack of immunological reagents (so abundant for the mouse) that makes it difficult to provide useful correlates of protection.

The final evaluation step is the primate model. Because of the expense and need for specialized facilities, this can only be used for the most promising candidates. At this time, there is still no consensus on which species of animal gives the best results (19).

There are several types of new candidate vaccines. At the time of writing, emphasis is centering on four types, 
namely subunit/fusion protein vaccines, DNA vaccines, recombinant $\mathrm{BCG}$ vaccines, and live-attenuated vaccines.

Subunit and fusion protein vaccines have been shown to protect in mice and to significantly prolong survival and reduce necrotic pathology in guinea pigs. Pooled proteins $(1,17,27)$, as well as individual proteins $(6,16,20)$, have been tested in various models, and several appear promising. Several laboratories have also derived polyproteins by gene fusion technology, and one of these designated 72f (Corixa, Seattle, WA, USA) has strong protective activity by itself and doubles the survival of guinea pigs when used as a BCG boost. Until recently, a major limitation to the use of this type of vaccine has been the need for an appropriate adjuvant, but over the past few years, adjuvants have slowly started to emerge that seem capable of driving the immune response to the candidate in a TH1 manner. These include various formulations based on monophosphoryl lipid A, the saponin QS21, and new synthetic adjuvants.

DNA vaccines are another area in which substantial progress has been made, with multiple targets. Vaccines against many of these seem to protect, at least in short-term assays (18). Enthusiasm for this approach waned for a period of time, because of concerns about safety and disappointing results in human trials, but seems to be returning in the form of "prime-boost" strategies. In these, DNA is given first, followed by protein antigens or antigens delivered in live vectors such as adenovirus. There are multiple variations on a theme here, with the boost given in adjuvant or as an adenovirus construct or other delivery devices, and it is probably too early to say how effective in the long run these approaches will be. Another question is whether the DNA will work if given as a boost following BCG. In our ongoing studies at CSU, DNA encoding 72f appears to be strongly boosting BCG in guinea pigs (Figure 1).

Recombinant BCG vaccines are also promising approaches to improve the efficacy of BCG. Recombinant BCG-secreting mammalian cytokines were an early idea in the field (22), but these induced more rapid clearance in animal models and reduced protection and have not been pursued further. A newer approach has been to overexpress key antigens in rBCG, and recent work (15) has shown that Ag85 can be overexpressed in this manner, resulting in a vaccine that appears to be more protective than BCG controls. Several candidates are under review at CSU at this time, but for a new BCG to be truly "better", we would like to see trustworthy evidence that the vaccine results in significantly better survival of guinea pigs. Since regular BCG protects these animals for at least one year, such studies are obviously very long. A third category of rBCG vaccines includes the elegant idea of expressing the gene for the lysin from Listeria in BCG (13). The idea here is that the listeriolysin molecule will allow the escape of mycobacterial antigens from the phagosome, promoting class-I presentation of antigens and a better CD8 T cell response. It will be very interesting to see if this strategy works, and results should be available from CSU at the end of the year.

A final area of interest regards the live-attenuated vaccines. This approach, pioneered by Jacobs, is an extension of early work in which several auxotrophic mutants were made from M. tuberculosis, with the idea being that they would vaccinate the host before they died and were cleared from the animal. These approaches have certainly worked (14), but more technical advances have now allowed "gene knockout" strategies to be used that can target specific genes. These include the RD1 region, the original set of genes that were lost by BCG, and the pantothenate metabolic pathway. Mutants lacking these genes immunize mice against virulent challenge but cannot themselves persist in the animal. Longer-term evaluations of these promising new candidates are currently underway.

\section{IMPACT OF PROTEOMICS/GENOMICS}

The publication of the $M$. tuberculosis genome by Cole et al. (9) provided a much needed instruction manual illustrating the genomic information possessed by $M$. tuberculosis. The genome contains nearly 4000 genes, a

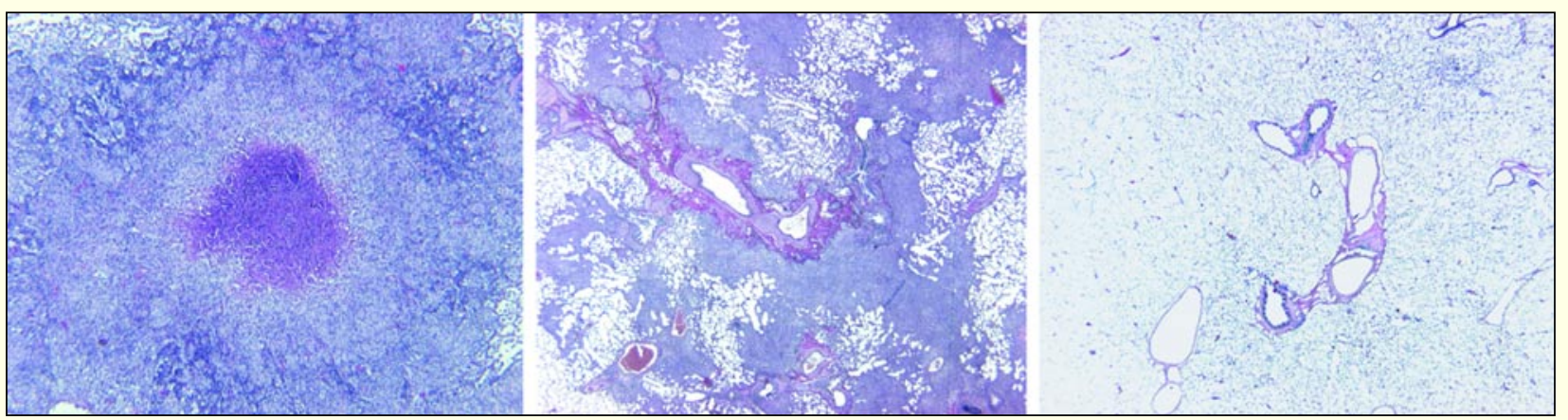

Figure 1. Light micrograph of lung tissue (stained with $\mathbf{H} \& \mathbf{E}$ ) of guinea pigs infected with $M$. tuberculosis. Infection of guinea pigs with $M$. tuberculosis results in a "classical" granuloma (left) with a prominent necrotic core (which will eventually mineralize) surrounded by a mantle of lymphocytes and macrophages embedded in a matrix of collagen. These lesions become very large and the animal dies after 15-20 weeks. Survival can be considerably prolonged by BCG vaccination (center). However, while the granuloma is now highly lymphocytic, it slowly invades much of the lung tissues, eventually severely consolidating the organ and killing the animal in 50-70 weeks. Recent studies using BCG boosted by polyproteins (right) prevents this effect, and most of the lesions resolve with only small areas of granulomatous tissue evident, usually around large vessels. The mechanism underlying this effect is currently completely unknown. 
large number of which seem to be involved in the biosynthesis of the highly complex bacterial cell wall. While this offers optimism in terms of identifying new drugs, in addition to the usual difficulties in drug development in terms of bioabsorption, pharmacokinetics, and toxicity, mycobacteria appear to be formidable drug targets.

Good progress is being made, however, in terms of using the genomic information to facilitate proteomic analyses. In a recent study (10), isoelectric focusing followed by gel electrophoresis was used to generate a 2-D separation of the proteins within the culture filtrate of $M$. tuberculosis, which is rich in immunogenic targets of the immune response (24). The protein spots are then partially digested and the fragments sequenced by mass spectroscopy (either MALDI-MS or the more accurate electron-spray MS/MS). From these sequences, many of the proteins can be identified as open reading frames in the genome using commercial programs such as SEQUEST. Some, such as the mycolyl transferase complex (3), can be identified by this process, although still about $50 \%$ of proteins continue to be of unknown function. By taking $\mathrm{T}$ cells from infected animals and culturing them with each protein and then looking for gamma interferon, one can prioritize these in terms of their relative immunogenicity.

\section{ACKNOWLEDGMENTS}

This work was supported by National Institutes of Health programs AI-45707 and AI-95320.

\section{REFERENCES}

1.Andersen, P. 1994. Effective vaccination of mice against Mycobacterium tuberculosis infection with a soluble mixture of secreted mycobacterial proteins. Infect. Immun. 62:2536-2544.

2.Baldwin, S.L., C. D'Souza, A.D. Roberts, B.P. Kelly, A.A. Frank, M.A. Lui, J.B. Ulmer, K. Huygen, et al. 1998. Evaluation of new vaccines in the mouse and guinea pig model of tuberculosis. Infect. Immun. 66:2951-2959.

3.Belisle, J.T., V.D. Vissa, T. Sievert, K. Takayama, P.J. Brennan, and G.S. Besra. 1997. Role of the major antigen of Mycobacterium tuberculosis in cell wall biogenesis. Science 276:1420-1422.

4.Behr, M.A. and P.M. Small. 1999. A historical and molecular phylogeny of BCG strains. Vaccine 17:915-922.

5.Behr, M.A., M.A. Wilson, W.P. Gill, H. Salamon, G.K. Schoolnik, S. Rane, and P.M. Small. 1999. Comparative genomics of BCG vaccines by whole-genome DNA microarray. Science 284:1520-1523.

6.Brandt, L., M. Elhay, I. Rosenkrands, E.B. Lindblad, and P. Andersen. 2000. ESAT-6 subunit vaccination against Mycobacterium tuberculosis. Infect. Immun. 68:791-795.

7.Brandt, L., J. Feino Cunha, A. Weinreich Olsen, B. Chilima, P. Hirsch, R. Appelberg, and P. Andersen. 2002. Failure of the $M y$ cobacterium bovis BCG vaccine: some species of environmental mycobacteria block multiplication of BCG and induction of protective immunity to tuberculosis. Infect. Immun. 70:672-678.

8.Colditz, G.A., T.F. Brewer, C.S. Berkey, M.E. Wilson, E. Burdick, H.V. Fineberg, and F. Mosteller. 1994. Efficacy of BCG vaccine in the prevention of tuberculosis. Meta-analysis of the published literature. JAMA 271:698-702.

9.Cole, S.T., R. Brosch, J. Parkhill, T. Garnier, C. Churcher, D. Harris, S.V. Gordon, K. Eiglmeier, et al. 1998. Deciphering the biology of Mycobacterium tuberculosis from the complete genome sequence. Nature 393:537-544.

10.Covert, B.A., J.S. Spencer, I.M. Orme, and J.T. Belisle. 2001. The application of proteomics in defining the T cell antigens of Mycobacterium tuberculosis. Proteomics 1:574-586.

11.Fine, P.E. 1989. The BCG story: lessons from the past and implications for the future. Rev. Infect. Dis. 11 Suppl 2:S353-S359.

12.Fine, P.E. 1995. Variation in protection by BCG: implications of and for heterologous immunity. Lancet 346:1339-1345.

13.Hess, J., D. Miko, A. Catic, V. Lehmensiek, D.G. Russell, and S.H. Kaufmann. 1998. Mycobacterium bovis Bacille Calmette-Guerin strains secreting listeriolysin of Listeria monocytogenes. Proc. Natl. Acad. Sci. USA 95:5299-5304.

14.Hondalus, M.K., S. Bardarov, R. Russell, J. Chan, W.R. Jacobs, Jr., and B.R. Bloom. 2000. Attenuation of and protection induced by a leucine auxotroph of Mycobacterium tuberculosis. Infect. Immun. 68:2888-2898.

15.Horwitz, M.A., G. Harth, B.J. Dillon, and S. Maslesa-Galic. 2000. Recombinant bacillus calmette-guerin (BCG) vaccines expressing the Mycobacterium tuberculosis 30-kDa major secretory protein induce greater protective immunity against tuberculosis than conventional BCG vaccines in a highly susceptible animal model. Proc. Natl. Acad. Sci. USA 97:13853-13858.

16.Horwitz, M.A., B.W. Lee, B.J. Dillon, and G. Harth. 1995. Protective immunity against tuberculosis induced by vaccination with major extracellular proteins of Mycobacterium tuberculosis. Proc. Natl. Acad. Sci. USA 92:1530-1534.

17.Hubbard, R.D., C.M. Flory, and F.M. Collins. 1992. Immunization of mice with mycobacterial culture filtrate proteins. Clin. Exp. Immunol. 87:94-98.

18.Huygen, K. 1998. DNA vaccines: application to tuberculosis. Int. J. Tuberc. Lung Dis. 2:971-978.

19.Langermans, J.A., P. Andersen, D. van Soolingen, R.A. Vervenne, P.A. Frost, T. van der Laan, L.A. van Pinxteren, J. van den Hombergh. 2001. Divergent effect of bacillus Calmette-Guerin (BCG) vaccination on Mycobacterium tuberculosis infection in highly related macaque species: implications for primate models in tuberculosis vaccine research. Proc. Natl. Acad. Sci. USA 98:11497-11502.

20.Leal, I. S., M. Florido, P. Andersen, and R. Appelberg. 2001. Interleukin-6 regulates the phenotype of the immune response to a tuberculosis subunit vaccine. Immunology 103:375-381.

21.McMurray, D.N. 1994. Guinea pig model of tuberculosis, p. 135147. In B.R. Bloom (Ed.), Tuberculosis: Pathogenesis, Protection, and Control. ASM Press, Washington D.C.

22.Murray, P.J., A. Aldovini, and R.A. Young. 1996. Manipulation and potentiation of antimycobacterial immunity using recombinant bacille Calmette-Guerin strains that secrete cytokines. Proc. Natl. Acad. Sci. USA 93:934-939.

23.Orme, I.M. 1999. Beyond BCG: the potential for a more effective TB vaccine. Mol. Med. Today 5:487-492.

24.Orme, I.M., P. Andersen, and W.H. Boom. 1993. T cell response to Mycobacterium tuberculosis. J. Infect. Dis. 167:1481-1497.

25.Orme, I.M. and F.M. Collins. 1985. Prophylactic effect in mice of BCG vaccination against nontuberculous mycobacterial infections. Tubercle 66:117-120.

26.Orme, I.M. and A.M. Cooper. 1999. Cytokine/chemokine cascades in immunity to tuberculosis. Immunol. Today 20:307-312.

27.Roberts, A.D., M.G. Sonnenberg, D.J. Ordway, S.K. Furney, P.J. Brennan, J.T. Belisle, and I.M. Orme. 1995. Characteristics of protective immunity engendered by vaccination of mice with purified culture filtrate protein antigens of Mycobacterium tuberculosis. Immunology 85:502-508.

Address correspondence to Dr. Ian Orme, Mycobacteria Research Laboratories, Dept. of Microbiology, Immunology and Pathology, Colorado State University, Fort Collins, CO 80523, USA.e-mail:iorme@lamar.colostate.edu 